\title{
The Jones slopes of a knot
}

\author{
Stavros Garoufalidis*
}

\begin{abstract}
The paper introduces the slope conjecture which relates the degree of the Jones polynomial of a knot and its parallels with the slopes of incompressible surfaces in the knot complement. More precisely, we introduce two knot invariants, the Jones slopes (a finite set of rational numbers) and the Jones period (a natural number) of a knot in 3-space. We formulate a number of conjectures for these invariants and verify them by explicit computations for the class of alternating knots, the knots with at most 9 crossings, the torus knots and the $(-2,3, n)$ pretzel knots.
\end{abstract}

Mathematics Subject Classification (2010). Primary 57N10; Secondary 57M25.

Keywords. Knot, link, Jones polynomial, Jones slope, Jones period, quasi-polynomial, alternating knots, signature, pretzel knots, polytopes, Newton polygon, incompressible surfaces, slope, slope conjecture.

\section{Introduction}

1.1. The degree of the Jones polynomial and incompressible surfaces. The paper introduces an explicit conjecture relating the degree of the Jones polynomial of a knot (and its parallels) with slopes of incompressible surfaces in the knot complement. We give an elementary proof of our conjecture for alternating knots and torus knots, and check it with explicit computations for non-alternating knots with 8 and 9 crossings, and for the $(-2,3, p)$ pretzel knots.

One side of our conjecture involves the growth rate of the degree $\delta_{K}(n)$ (with respect to $q$ ) of the colored Jones function $J_{K, n}(q) \in \mathbb{Z}\left[q^{ \pm 1}\right]$ of a knot. The other side involves the finite set $\mathrm{bs}_{K}$ of slopes of incompressible, $\partial$-incompressible orientable surfaces in the complement of $K$, where the slopes are normalized so that the longitude has slope 0 and the meridian has slope $\infty$; [Ha]. To formulate our conjecture, we need a definition. Recall that $x \in \mathbb{R}$ is a cluster point of a sequence $\left(x_{n}\right)$ of real numbers if for every $\varepsilon>0$ there are infinitely many indices $n \in \mathbb{N}$ such that $\left|x-x_{n}\right|<\varepsilon$. Let $\left\{x_{n}\right\}^{\prime}$ denote the set of cluster points of a sequence $\left(x_{n}\right)$.

\footnotetext{
${ }^{*}$ The author was supported in part by NSF.
} 
Definition 1.1. (a) For a knot $K$, define the Jones slopes $\mathrm{js}_{K}$ by

$$
\mathrm{js}_{K}=\left\{\frac{2}{n^{2}} \operatorname{deg}\left(J_{K, n}(q)\right) \mid n \in \mathbb{N}\right\}^{\prime} .
$$

(b) Let bs $_{K}$ denote the set of boundary slopes of incompressible surfaces of $K$.

A priori, the structure and the cardinality of the set $\mathrm{js}_{K}$ is not obvious. On the other hand, it is known that $b_{K}$ is a finite subset of $\mathbb{Q} \cup\{\infty\}$; see [Ha]. Normal surfaces are of special interest because of their relation with exceptional Dehn surgery, and the $\operatorname{SL}(2, \mathbb{C})$ character variety and hyperbolic geometry, see for example [Bu], [CGLS], [CCGLS], [KR], [LTi], [Mv].

Conjecture 1 (The slope conjecture). For every knot we have

$$
2 \mathrm{js}_{K} \subset \mathrm{bs}_{K} \text {. }
$$

Before we proceed further, and to get a better intuition about this conjecture, let us give three illustrative examples.

Example 1.2. For the alternating knot $8_{17}$ we have

$$
\delta(n)=2 n^{2}+2 n, \quad \delta^{*}(n)=-2 n^{2}-2 n,
$$

where $\delta_{K}(n)$ and $\delta_{K}^{*}(n)$ are the maximum and the minimum degree of $J_{K, n}(q)$ with respect to $q$. On the other hand, according to $[\mathrm{Cu}]$, the Newton polygon (based on the geometric component of the character variety) has 44 sides and its slopes (excluding multiplicities) are

$$
\{-14,-8,-6,-4,-2,0,2,4,6,8,14, \infty\} \text {. }
$$

The reader may observe that $\delta(n)$ and $\delta^{*}(n)$ are quadratic polynomials in $n$ and four times the leading terms of $\delta(n)$ and $\delta^{*}(n)$ are boundary slopes (namely 8 and -8 ), and moreover they agree with $2 c^{+}$and $-2 c^{-}$where $c^{ \pm}$is the number of positive/negative crossings of $8_{17}$. In addition, as Y. Kabaya observed (see [Ka]), $8_{17}$ has slopes \pm 14 outside the interval $\left[-2 c^{-}, 2 c^{+}\right]=[-8,8]$.

Example 1.3. For the non-alternating pretzel knot $(-2,3,7)$ we have

$$
\delta(n)=\left[\frac{37}{8} n^{2}+\frac{17}{2} n\right]=\frac{37}{8} n^{2}+\frac{17}{2} n+\varepsilon(n), \quad \delta^{*}(n)=5 n,
$$

where $\varepsilon(n)$ is a periodic sequence of period 4 given by $0,1 / 8,1 / 2,1 / 8$ if $n \equiv$ $0,1,2,3 \bmod 4$ respectively. $(-2,3,7)$ is a Montesinos knot and its boundary slopes are given by

$$
\left\{0,16, \frac{37}{2}, 20\right\}
$$

(see $[\mathrm{HO}]$ and $[\mathrm{Du}]$ and compare also with [Ma]). In this case, $\delta(n)$ is no longer a quadratic polynomial of $n$. Instead $\delta(n)$ is a quadratic quasi-polynomial with fixed 
leading term $37 / 8$. Moreover, four times this leading term is a slope of the knot. This number was the motivating example that eventually lead to the results of this paper. Likewise, four times the $n^{2}$-coefficient of $\delta^{*}(n)$ is 0 , which is also a boundary slope of this knot.

Example 1.4. The pretzel knots $(2,3,5,5)$ and $(2,5,3,5)$ are mutant, alternating and Montesinos. Since they are mutant, their colored Jones functions (thus their Jones slopes) agree; see [Kf]. Since they are alternating, their common Jones slopes are js $=c^{+}=15$ and $\mathrm{js}^{*}=-c^{-}=0$; see Theorem 2. Since they are Montesinos, Hatcher-Oertel's algorithm implemented by Dunfield (see [Du]) implies that their boundary slopes are given by

$$
\begin{aligned}
& \operatorname{bs}_{(2,3,5,5)}=\{0,4,6,10,14,16,18,20,22,24,26,28,30, \infty\}, \\
& \operatorname{bs}_{(2,5,3,5)}=\{0,4,6,10,14,16,18,20,22,26,28,30, \infty\} .
\end{aligned}
$$

It follows that the set $\mathrm{bs}_{K}$ is not invariant under knot mutation.

\subsection{The degree of the colored Jones function is a quadratic quasi-polynomial.} In the previous section, we took the shortest path to formulate a conjecture relating the degree of the colored Jones function of a knot with incompressible surfaces in the knot complement. In this section we will motivate our conjecture, and add some structure to it. Let us recall that in 1985 Jones introduced the famous Jones polynomial $J_{K}(q) \in \mathbb{Z}\left[q^{ \pm 1}\right]$ of a knot (or link) $K$ in 3-space; [Jo]. The Jones polynomial of a knot is a Laurent polynomial with integer coefficients that tightly encodes information about the topology and the geometry of the knot.

Unlike the Alexander polynomial, not much is known about the topological meaning of the coefficients of the Jones polynomial, nor about its degree, nor about the countable set of Jones polynomials of knots.

This unstructured behavior of the Jones polynomial becomes more structured when one fixes a knot $K$ and considers a stronger invariant, namely the colored Jones function $J_{K, n}(q)$. The latter is a sequence of elements of $\mathbb{Z}\left[q^{ \pm 1}\right]$ indexed by $n \in \mathbb{N}$ which encodes the TQFT invariants of a knot colored by the irreducible $(n+1)$-dimensional representation of SU(2), and normalized to be 1 at the unknot; see [Tu2]. With these conventions, $J_{K, 0}(q)=1$ and $J_{K, 1}(q)$ is the Jones polynomial of $K$.

In many ways the sequence $J_{K, n}(q)$ is better behaved and suitable limits of the sequence $J_{K, n}(q)$ have a clear topological or geometric meaning. Let us give three instances of this phenomenon:

(a) a suitable formal power series limit $J_{K, n-1}\left(e^{h}\right) \in \mathbb{Q}[[h, n]]$ (known as the Melvin-Morton-Rozansky conjecture) equals to $1 / \Delta_{K}\left(e^{n h}\right)$ and determines the Alexander polynomial $\Delta_{K}(t)$ of $K$ (see [B-NG]);

(b) an analytic limit $J_{K, N-1}\left(e^{\alpha / N}\right)$ for small complex numbers $\alpha$ near zero equals to $1 / \Delta_{K}\left(e^{\alpha}\right)$ also determines the Alexander polynomial of $K$; see [GL1], Thm. 2; 
(c) the exponential growth rate of the sequence $J_{K, N-1}\left(e^{2 \pi i / N}\right)$ (the so-called Kashaev invariant) of a hyperbolic knot is conjectured to equal to the volume of $K$ divided by $2 \pi$; see [Ks].

On the other hand, one can easily construct hyperbolic knots with equal Jones polynomial but different Alexander polynomial and volume.

Some already observed structure regarding the colored Jones function $J_{K, n}(q)$ is that it is $q$-holonomic, i.e., it satisfies a linear recursion relation with coefficients in $\mathbb{Z}\left[q^{n}, q\right]$; see [GL1]. The present paper is concerned with another notion of regularity, namely the degree of the colored Jones function $J_{K, n}(q)$ with respect to $n$. Since little is known about the degree of the Jones polynomial of a knot, one might expect that there is little to say about the degree of the colored Jones function $J_{K, n}(q)$. Once observed, the regularity of the degree seems obvious as Bar-Natan suggests; see [B-NL], Lemma 3.6, and [Me]. Moreover, the degree of the colored Jones function motivates the introduction of two knot invariants, the Jones slopes of a knot (a finite set of rational numbers) and the Jones period of a knot (a natural number).

1.3. $q$-holonomic functions and quadratic quasi-polynomials. To formulate our new notion of regularity, we need to recall what is a quasi-polynomial. A quasipolynomial $p(n)$ is a function

$$
p: \mathbb{N} \rightarrow \mathbb{N}, \quad p(n)=\sum_{j=0}^{d} c_{j}(n) n^{j},
$$

for some $d \in \mathbb{N}$ where $c_{j}(n)$ is a periodic function with integral period for $j=$ $1, \ldots, d$; [St], [BR]. If $c_{d}(n)$ is not identically zero, then the degree of $p$ is $d$. We will focus on two numerical invariants of a quasi-polynomial, its period and its slopes.

Definition 1.5. (a) The period $\pi$ of a quasi-polynomial $p(n)$ as above is the common period of $c_{j}(n)$.

(b) The set of slopes of a quadratic quasi-polynomial $p(n)$ is the finite set of twice the rational values of the periodic function $c_{2}(n)$.

Notice that if $p(n)$ is a quasi-polynomial of period $\pi$, then there exist polynomials $p_{0}, \ldots, p_{s-1}$ such that $p(n)=p_{i}(n)$ when $n \equiv i \bmod \pi$, and vice versa. Notice also that the set of slopes of a quasi-polynomial is always a finite subset of $\mathbb{Q}$.

Quasi-polynomials of period 1 are simply polynomials. Quasi-polynomials appear naturally in counting problems of lattice points in rational convex polytopes; see for example [BP], [BR], [BV], [Eh], [St]. In fact, if $P$ is a rational convex polytope, then the number of lattice points of $n P$ is the so-called Ehrhart quasi-polynomial of $P$, useful in many enumerative questions [BP], [BR], [BV], [Eh], [St].

The next theorem seems obvious, once observed. The proof, given in [Ga2], uses ideas from differential Galois theory of $D$-modules and the key Skolem-Mahler-Lech theorem from number theory. Let $\operatorname{deg}(f(q))$ denote the degree of a rational function $f(q)$ with respect to $q$. 
Theorem 1 ([Ga2]). If $f_{n}(q)$ is a q-holonomic sequence of rational functions, then $\operatorname{deg}\left(f_{n}(q)\right)$ is a quadratic quasi-polynomial for large $n$. Moreover, the leading term of $f_{n}(q)$ satisfies a linear recursion relation with constant coefficients.

The restriction for large $n$ in Theorem 1 is necessary, since the sequence $((1+$ $\left.\left.(-1)^{n}\right) q^{n^{2}}+q^{17}\right)$ is $q$-holonomic, and its degree (given by 17 if $n \leq 4$, by $n^{2}$ if $n \geq 5$ is even and by 17 if $n \geq 5$ is odd) is not a quasi-polynomial. On the other hand, if $n \geq 5$, the degree is given by the quadratic quasi-polynomial $n^{2}\left(1+(-1)^{n}\right) / 2+17$.

Corollary 1.6. If $f_{n}(q)$ is a $q$-holonomic sequence of rational functions and $\delta(n)=$ $\operatorname{deg}\left(f_{n}(q)\right)$ then

$$
\left\{\frac{2}{n^{2}} \delta(n) \mid n \in \mathbb{N}\right\}^{\prime}=\operatorname{slopes}(\delta) .
$$

Proof. Consider a subsequence $k_{n}$ of the natural numbers such that

$$
l=\lim _{n} 2 \frac{\delta\left(k_{n}\right)}{k_{n}^{2}} .
$$

Since $d(n)$ is (for large $n$ ) given by a quasi-polynomial, it follows that $d(n)=$ $c_{2}(n) n^{2}+O(n)$ for a periodic function $c_{2}: \mathbb{N} \rightarrow \mathbb{Q}$ and for all $n \in \mathbb{N}$. Since $c_{2}$ takes finitely many values, it follows that there is a subsequence $m_{n}$ of $k_{n}$ such that $c_{2}\left(m_{n}\right)=s$ for all $n$, where $s$ is a slope of $\delta(n)$. Since $d(n)=c_{2}(n) n^{2}+O(n)$ for all $n$, it follows that $l=2 s$, i.e., $l$ is a slope of $\delta$. Conversely, it is easy to see that every slope of $\delta$ is the limit point for a subsequence taken to be an arithmetic progression on which $c_{2}$ takes a constant value.

1.4. The Jones slopes and the Jones period of a knot. Given a knot $K$, we set

$$
\delta_{K}(n)=\operatorname{deg}\left(J_{K, n}(q)\right) .
$$

Combining the $q$-holonomicity of the colored Jones function $J_{K, n}(q)$ of a knot $K$ with Theorem 1, it follows that $\delta_{K}$ is a quadratic quasi-polynomial for large $n$.

Definition 1.7. (a) The Jones period $\pi_{K}$ is the period of $\delta_{K}$.

(b) The Jones slopes js $_{K}$ is the finite set of slopes of $\delta_{K}$.

Corollary 1.6 implies that for every knot $K$ we have

$$
\left\{\frac{2}{n^{2}} \operatorname{deg}\left(J_{K, n}(q)\right) \mid n \in \mathbb{N}\right\}^{\prime}=\operatorname{slopes}\left(\delta_{K}\right),
$$

where the right-hand side (and consequently, the left-hand side, too) is a finite subset of $\mathbb{Q}$.

Lemma 1.8. For every knot $K$ we have

$$
\left\{\frac{2}{n^{2}} \operatorname{deg}\left(J_{K, n}(q)\right) \mid n \in \mathbb{N}\right\}^{\prime}=\operatorname{slopes}\left(\delta_{K}\right),
$$

where slopes $\left(\delta_{K}\right)$ is a finite subset of $\mathbb{Q}$. 
The $\delta_{K}$ invariant records the growth rates of the (maximum) degree of the colored Jones function of $K$. We can also record the minimum degree as follows. If $K^{*}$ denotes the mirror image of $K$, then $J_{K^{*}, n}(q)=J_{K}\left(q^{-1}\right)$. Let us define

$$
\delta_{K}^{*}(n)=-\delta_{K^{*}}(n)=\operatorname{mindeg}\left(J_{K, n}(q)\right), \quad \mathrm{js}_{K}^{*}=\operatorname{slopes}\left(\delta_{K}^{*}\right) .
$$

Notice that Conjecture 1 applied to $K^{*}$ implies that

$$
\mathrm{js}_{K}^{*} \subset \mathrm{bs}_{K}
$$

The next proposition gives a bound for the Jones slopes of a knot $K$ in terms of the number $c_{K}^{ \pm}$of positive/negative crossings of a planar projection.

Proposition 1.9. For every knot $K$, every $s \in \mathrm{js}_{K}$ and every $s^{*} \in \mathrm{js}_{K}^{*}$ we have

$$
-c_{K}^{-} \leq s^{*}, s \leq c_{K}^{+}
$$

The reader may compare the above lemma with Example 1.2.

Our next theorem confirms Conjecture 1 for all alternating knots. Consider a reduced planar projection of $K$ with $c_{K}^{ \pm}$crossings of positive/negative sign.

Theorem 2. If $K$ is alternating, then

$$
\pi_{K}=1, \quad \mathrm{js}_{K}=\left\{c_{K}^{+}\right\}, \quad \mathrm{js}_{K}^{*}=\left\{-c_{K}^{-}\right\} .
$$

In addition, the two checkerboard surfaces of $K$ (doubled, if need, to make them orientable) are incompressible with slopes $2 c_{K}^{+}$and $-2 c_{K}^{-}$.

Our final lemma relates the Jones slopes and the period of a knot.

Lemma 1.10. If $a(n)$ is an integer-valued quadratic quasi-polynomial with period $\pi$, then for every slope s of $a(n)$ we have

$$
s \pi^{2} \in \mathbb{Z}
$$

In particular, for every knot $K$ we have

$$
\pi_{K}^{2} \mathrm{js}_{K} \subset \mathbb{Z}, \quad \pi_{K}^{2} \mathrm{js}_{K}^{*} \subset \mathbb{Z} .
$$

Thus, if a knot has a non-integral Jones slope, then it has period bigger than 1.

1.5. The symmetrized Jones slopes and the signature of a knot. In this section we discuss the symmetrized version $\delta_{K}^{ \pm}$of $\delta_{K}$ :

$$
\delta_{K}^{+}=\delta_{K}-\delta_{K}^{*}, \quad \delta_{K}^{-}=\delta_{K}+\delta_{K}^{*}
$$

Of course, $\delta_{K}=1 / 2\left(\delta_{K}^{+}+\delta_{K}^{-}\right)$and $\delta_{K}^{*}=1 / 2\left(-\delta_{K}^{+}+\delta_{K}^{-}\right)$. Pictorially, we have 


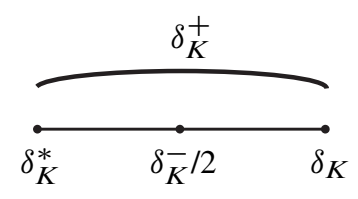

As we will see below, the symmetrized degree $\delta_{K}^{ \pm}$of the colored Jones function has a different flavor, and relates (at least for alternating knots) to the signature of the knot. $\delta_{K}^{+}(n)$ is the span of $J_{K, n}(q)$, i.e., the difference between the maximum and minimum degree of $J_{K, n}(q)$. On the other hand, $\delta_{K}^{-}(n)$ is the sum of the minimum and maximum degree of $J_{K, n}(q)$, and appears to be less studied. Of course, $\delta_{K}^{ \pm}$are quadratic quasi-polynomials. Since the colored Jones function is multiplicative under connected sum, and reverses $q$ to $q^{-1}$ under mirror image, it follows that

$$
\delta_{K_{1} \# K_{2}}^{-}=\delta_{K_{1}}^{-}+\delta_{K_{2}}^{-}, \quad \delta_{K^{*}}^{-}=-\delta_{K}^{-} .
$$

Our next theorem computes the $\delta_{K}^{ \pm}$quasi-polynomials of an alternating knot $K$ in terms of three basic invariants: the signature $\sigma_{K}$, the writhe $w_{K}$ and the number of crossings $c_{K}$ of a reduced projection of $K$. Our result follows from elementary linear algebra using the results of Kauffman, Murasugi and Thistlethwaite, [Kf], [Mu], [Th], further simplified by Turaev [Tu1]. See also [Li], p. 42, and [Le], Prop. 2.1.

Theorem 3. (a) For all alternating knots $K$ we have

$$
\begin{aligned}
& \delta_{K}^{-}(n)=\frac{w_{K}}{2} n^{2}+\frac{w_{K}-2 \sigma_{K}}{2} n, \\
& \delta_{K}^{+}(n)=\frac{c_{K}}{2} n^{2}+\frac{c_{K}}{2} n .
\end{aligned}
$$

(b) The Jones polynomial $J_{K}(q)$ determines $c_{K}$ by

$$
\delta_{K}^{+}(1)=c_{K} .
$$

(c) The Jones polynomial of $K$ and its 2-parallel determines $w_{K}$ and $\sigma_{K}$ by

$$
\sigma_{K}=-3 \delta_{K}^{-}(1)+\delta_{K}^{-}(2), \quad w_{K}=-2 \delta_{K}^{-}(1)+\delta_{K}^{-}(2) .
$$

Remark 1.11. Part (c) of Theorem 3 is sharp. The Jones polynomial of an alternating knot determines the number of crossings, but it does not determine the signature nor the writhe of the knot. Shumakovitch provided us with a table of pairs of alternating knots with up to 14 crossings (using the Thistlethwaite notation) with equal Jones polynomials and unequal signature. An example of such a pair with 12 crossings is the knot $12 a_{669}$ and its mirror image: 


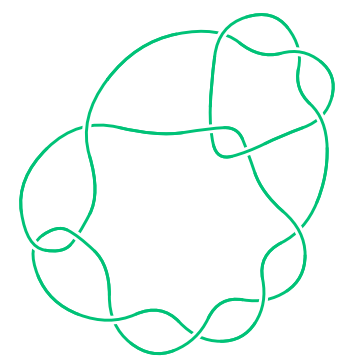

$12 a_{669}$ has Jones polynomial

$J_{12 a_{669}, 1}(q)=-\frac{1}{q^{6}}+\frac{2}{q^{5}}-\frac{4}{q^{4}}+\frac{6}{q^{3}}-\frac{7}{q^{2}}+\frac{9}{q}-9+9 q-7 q^{2}+6 q^{3}-4 q^{4}+2 q^{5}-q^{6}$

and signature -2 . Since the signature is nonzero, $12 a_{669}$ is not amphicheiral, and yet has palindromic Jones polynomial. The next colored Jones polynomial is given by

$$
\begin{aligned}
J_{12 a_{669}, 2}(q)= & \frac{1}{q^{17}}-\frac{2}{q^{16}}+\frac{1}{q^{15}}+\frac{3}{q^{14}}-\frac{7}{q^{13}}+\frac{4}{q^{12}}+\frac{6}{q^{11}}-\frac{13}{q^{10}}+\frac{6}{q^{9}}+\frac{8}{q^{8}} \\
& -\frac{15}{q^{7}}+\frac{7}{q^{6}}+\frac{7}{q^{5}}-\frac{15}{q^{4}}+\frac{11}{q^{3}}+\frac{6}{q^{2}}-\frac{21}{q}+18+9 q-30 q^{2}+20 q^{3} \\
& +15 q^{4}-35 q^{5}+16 q^{6}+20 q^{7}-32 q^{8}+7 q^{9}+22 q^{10}-22 q^{11} \\
& -2 q^{12}+17 q^{13}-9 q^{14}-5 q^{15}+7 q^{16}-q^{17}-2 q^{18}+q^{19}
\end{aligned}
$$

and is far from being palindromic. This is another example where the pattern of the Jones polynomial is blurred, but the pattern of the colored Jones function is clearer.

Results similar to Theorems 2 and 3 have been also been obtained independently in $[\mathrm{CT}]$ using the Jones polynomial of an alternating knot.

Let us end this section with two comments. In this paper $K$ is a knot, but without additional effort one can state similar results for a link (and even a knotted trivalent graph, or quantum spin network) in 3-space. In addition, we should point out that there are deeper aspects of stability and integrality of the coefficients of the colored Jones function. We will discuss them in a future publication.

1.6. Plan of the proof. In Section 3 we use the Kauffman bracket skein module and the work of Kauffman, Le, Murasugi Thistlethwaite to give a proof of Proposition 1.9 and Theorems 2 and 3. This proves our slope conjecture for all alternating knots.

In Section 4 we give computational evidence for the degree of the colored Jones polynomials (and for the slope conjecture) of the non-alternating knots with 8 and 9 crossings. In addition, we verify the slope conjecture for all $(-2,3, p)$ pretzel knots (using the fusion state-sum formulas of the colored Jones function studied in [GL], [Co], [GVa]), and all torus knots (using Morton's formula for the colored Jones function of those, $[\mathrm{Mo}])$. The reader may note that the pretzel knots $(-2,3, p)$ 
are well-known examples of Montesinos knots, with non-integral slopes (for $p \neq$ $-1,1,3,5)$, which are not quasi-alternating, thus the results of [FKP] do not apply for this family.

\section{Future directions}

In this section we will discuss some future directions, and pose some questions, problems and conjectures.

The slope conjecture involves knots in 3-space, and relates the degree of the colored Jones function to their set of boundary slopes. The slope conjecture may be extended in three different directions: one may consider (a) links in 3-space, (b) general 1-cusped manifolds and (c) general Lie algebras. These extensions have been considered by the author. In [GVu] we introduce a slope conjecture for knots and arbitrary Lie algebras. One may also consider 1-cusped manifolds with the homology of $S^{1} \times D^{2}$, i.e., knot complements in integer homology spheres. For those, the colored Jones polynomial exists (though it is not a polynomial, but rather an element of the Habiro ring; see [GL2]). The colored Jones function is $q$-holonomic, and we can use the non-commutative $A$-polynomial of this sequence to define the set of slopes.

The following problem appears mysterious and tantalizing.

Problem 1. Understand the selection principle which selects

(a) the Jones slope from the set of boundary slopes,

(b) the colored Jones function from the vector space of solutions of the linear $q$ difference equation.

Now let us post some questions, based on the limited experimental evidence. Several authors have studied the diameter $d_{K}$ of the set bs $s_{K}$

$$
d_{K}=\max \left\{\left|s-s^{\prime}\right| \mid s, s^{\prime} \in \operatorname{bs}_{K}\right\} .
$$

See for example [IM1], [IM2], [MMR]. Y. Kabaya pointed out to us that there are alternating knots $K$ with diameter bigger than twice the number of crossings; see Example 1.2. Let $\mathrm{jd}_{K}$ denote the Jones diameter

$$
\mathrm{jd}_{K}=\max \left\{\left|s-s^{*}\right| \mid s \in \mathrm{js}_{K}, s^{*} \in \mathrm{js}_{K}^{*}\right\} .
$$

Proposition 1.9 shows that $\mathrm{jd}_{K} \leq c_{K}$. Moreover, the bound is achieved for alternating knots, and more generally for adequate knots; see [FKP]. The next question concerns the class of knots of maximal Jones diameter.

Question 1. Is it true that a prime knot $K$ is adequate if and only if $\mathrm{jd}_{K}=c_{K}$ ? 
The class of alternating knots is included in two natural classes: quasi-alternating knots, and adequate knots. Knot Homology (and its exact triangles) can tell whether a knot is quasi-alternating or not (see [MO]), but it seems hard to tell whether a knot is alternating or not. Adequate knots appeared in [LT] in relation to the Jones polynomial and also in [FKP]. It was pointed out to us by D. Futer, E. Kalfagianni, J. Purcell and P. Ozsváth that the pretzel knots $(-2,3, p)$ are not adequate nor quasi-alternating for $p>5$.

In all examples, the set $\mathrm{js}_{K}$ consists of a single element, whereas the set $\mathrm{bs}_{K}$ can have arbitrarily many elements. Thus, Conjecture 1 sees only a small part of the set $\operatorname{bs}_{K}$. Our next conjecture claims that the colored Jones function $J_{K, n}(q)$ of $K$ may see all the elements in $\operatorname{bs}_{K}$. To formulate it, recall that $J_{K, n}(q)$ is a $q$-holonomic sequence, and satisfies a unique, minimal order recursion relation of the form

$$
\sum_{k=0}^{d} a_{k}\left(q^{n}, q\right) J_{K, n+k}(q)=0,
$$

where $a_{k}[u, v] \in \mathbb{Q}[u, v]$ are polynomials with greatest common divisor 1 ; see [Ga1]. The 3-variable polynomial $q A_{K}(E, Q, q)=\sum_{k=0}^{d} a_{k}(Q, q) E^{k}$ is often called the non-commutative $A$-polynomial of $K$. The AJ Conjecture of [Ga1] states that every irreducible factor of $q A_{K}(L, M, 1)$ is a factor of $A_{K}\left(L, M^{2}\right)$ or is $L$-free, and viceversa. Here $A_{K}$ denotes the $A$-polynomial of $K$ and $A_{K}$ contains all components of the $\operatorname{SL}(2, \mathbb{C})$ character variety of $K$ (including the abelian one). Let $\mathrm{bs}_{K}^{A}$ denote the slopes of the Newton polygon of $A$. These are the so-called visible slopes of a knot. It follows by Culler-Shalen theory (see [CS], [CGLS], [CCGLS]) that bs ${ }_{K}^{A} \subset \mathrm{bs}_{K}$.

Let us define the $q$-Newton polytope $q N_{K}$ of $K$ to be the convex hull of the monomials $q^{c} Q^{b} E^{a}$ of $q A_{K}(E, Q, q)$. Then $q N$ is a convex polytope in $\mathbb{R}^{3}$, and we may consider the image of it in $\mathbb{R}^{2}$ under the projection map $\mathbb{R}^{3} \rightarrow \mathbb{R}^{2}$ which maps $(a, b, c)$ to $(a, b)$ (i.e., sends the monomial $q^{c} Q^{b} E^{a}$ to $\left.Q^{b} E^{a}\right)$.

Definition 2.1. The $q$-slopes $\mathrm{qs}_{K}$ of a knot $K$ are the slopes of the projection of $q N$ to $\mathbb{R}^{2}$.

Problem 2. Show that for every knot $K$ we have

$$
2 \mathrm{qs}_{K}=\mathrm{bs}_{K}^{A} \text {. }
$$

It is easy to see that for every knot $K$ we have js ${ }_{K} \subset \mathrm{qs}_{K}$. In fact, this holds for arbitrary $q$-holonomic sequences; see [Ga4], Prop. 1.2, for a detailed discussion. Thus, Problem 2 implies Conjecture 1. The AJ Conjecture motivates Problem 2. This is discussed in detail in [Ga4].

Our next problem concerns the symmetrized quasi-polynomial $\delta^{-}$of a knot from (7). Although $\delta^{-}$is not a concordance invariant, it determines the signature of an alternating knot.

Problem 3. Show that $\delta^{-}$determines a Knot Homology invariant. 


\section{The Jones slopes and the Jones period of an alternating knot}

In this Section we prove Proposition 1.9 and Theorems 2 and 3 for an alternating knot $K$, using the Kauffman bracket presentation of the colored Jones polynomial.

The following lemma of Lê [Le], Prop. 2.1, (based on well-known properties of the Kauffman bracket skein module) shows that the sequences $\delta_{K}^{*}(n)$ and $\delta_{K}(n)$ have at most quadratic growth rate with respect to $n$. More precisely, for every knot $K$ we have:

$$
-\frac{1}{2} c_{K}^{-} n^{2}+O(n) \leq \delta_{K}^{*}(n) \leq \delta_{K}(n) \leq \frac{1}{2} c_{K}^{+} n^{2}+O(n) .
$$

This implies that the slopes $s$ of the quadratic quasi-polynomial $\delta_{K}$ satisfy $-c_{K}^{-} \leq$ $s \leq c_{K}^{+}$. Replacing $K$ by its mirror $K^{*}$, it implies the same inequality for the slopes $s^{*}$ of $\delta_{K}^{*}$ and concludes the proof of Proposition 1.9.

Consider a reduced planar projection of an alternating knot $K$ with $c_{K}^{ \pm}$positive/negative crossings. Then, the number of crossings $c_{K}$ and the writhe $w_{K}$ of $K$ are given by $c_{K}=c_{K}^{+}+c_{K}^{-}$and $w_{K}=c_{K}^{+}-c_{K}^{-}$. Let $\sigma_{K}$ denote the signature of $K$. Then we can express the minimum and maximum degrees $\delta_{K}^{*}(n)$ and $\delta_{K}(n)$ of $K$ in terms of $w_{K}, c_{K}$ and $\sigma_{K}$. This was shown by Kauffman, Murasugi and Thistlethwaite, [Kf], [Mu], [Th], and further simplified by Turaev [Tu1]. See also [Li], p. 42, and [Le], Prop. 2.1. With our conventions, Proposition 2.1 of [Le] states that for all $n$ we have

$$
\begin{aligned}
& \delta_{K}(n)=\frac{\left(c_{K}+w_{K}\right)}{4} n^{2}+\frac{-|A|+2 c_{K}^{+}+1}{2} n, \\
& \delta_{K}^{*}(n)=\frac{\left(-c_{K}+w_{K}\right)}{4} n^{2}+\frac{|B|-2 c_{K}^{-}-1}{2} n,
\end{aligned}
$$

where $|A|$ (resp. $|B|$ ) is the number of circles of the $A$ (resp. $B$ ) smoothing of the planar projection. For example, for the right-handed trefoil $T$, we have

$$
\begin{aligned}
J_{T, 0}(q)= & 1, \\
J_{T, 1}(q)= & q+q^{3}-q^{4}, \\
J_{T, 2}(q)= & q^{2}+q^{5}-q^{7}+q^{8}-q^{9}-q^{10}+q^{11}, \\
J_{T, 3}(q)= & q^{3}+q^{7}-q^{10}+q^{11}-q^{13}-q^{14}+q^{15}-q^{17}+q^{19}+q^{20}-q^{21}, \\
J_{T, 4}(q)= & q^{4}+q^{9}-q^{13}+q^{14}-q^{17}-q^{18}+q^{19}-q^{22}-q^{23}+2 q^{24} \\
& \quad-q^{28}+2 q^{29}-q^{32}-q^{33}+q^{34}
\end{aligned}
$$

and

$$
\begin{array}{ll}
\delta_{T}(n)=\frac{3}{2} n^{2}+\frac{5}{2} n, & \delta_{T}^{*}(n)=n, \\
\delta_{T}^{+}(n)=\frac{3}{2} n^{2}+\frac{3}{2} n, & \delta_{T}^{-}(n)=\frac{3}{2} n^{2}+\frac{7}{2} n
\end{array}
$$


and

$$
c_{T}^{+}=3, \quad c_{T}^{-}=0, \quad c_{T}=3, \quad w_{T}=3, \quad \sigma_{T}=-2, \quad|A|=2, \quad|B|=3 .
$$

Murasugi and Turaev observe that [Tu1], p. 219-220,

$$
\begin{aligned}
|A|+|B| & =c_{K}+2, \\
c_{K} & =c_{K}^{+}+c_{K}^{-}, \\
w_{K} & =c_{K}^{+}-c_{K}^{-}, \\
\sigma_{K} & =|A|-1-c_{K}^{+}=-|B|+1+c_{K}^{-} .
\end{aligned}
$$

Equation (17) implies that $\delta_{K}(n)$ is a quadratic polynomial (i.e., a quasi-polynomial of period 1) with coefficient of $n^{2}$ equal to $c_{K}^{+} / 2$, i.e., with slope $c_{K}^{+}$. This concludes the proof of Theorem 2. Equations (7), (16), (17) and (18) prove the first part of Theorem 3.

It remains to show that the two checkerboard surfaces of a reduced projection of an alternating knot $K$ have slopes $2 c_{K}^{+}$and $-2 c_{K}^{-}$. Observe that if $s=p m+q l$ is the slope of a surface $S$ (where $(m, l)$ is the standard meridian-longitude pair) and $\langle\cdot, \cdot\rangle$ denotes the form in the boundary of a neighborhood of $K$, then $q=\langle m, s\rangle$, $p=\langle s, l\rangle$. If $S$ is a black surface with slope $s=p m+q l$, then the geometrically $s$ and $m$ intersect at a point, thus $q= \pm 1$. In addition, $s$ follows the knot $K$ as we move towards the crossing, and intersects $l$ twice around each positive crossing, and none around each negative crossing. The result follows.

Remark 3.1. Let $V$ denote the 3-dimensional $\mathbb{Q}$-vector space spanned by the functions $c, w, \sigma$ on the set of alternating knots. There is an involution $K \mapsto K^{*}$ on this set, which includes an involution on $V$ :

$$
c^{*}=c, \quad w^{*}=-w, \quad \sigma^{*}=-\sigma .
$$

On the other hand, $\delta, \delta^{*}$ and $\delta^{ \pm}$belong to $V$ and

$$
\left(\delta^{+}\right)^{*}=\delta^{+}, \quad\left(\delta^{-}\right)^{*}=-\delta^{-} .
$$

Thus, $\delta^{+}$is a $\mathbb{Q}$-linear combination of $c$, and $\delta^{-}$is a $\mathbb{Q}$-linear combination of $w$ and $\sigma$. This is precisely the content of Theorem 3 .

Remark 3.2. Let $f[k]$ denote the coefficient of $n^{k}$ in a polynomial $f(n)$. Equations (9) and (10) imply that for all alternating knots $K$ we have

$$
c_{K}=2 \delta_{K}^{+}[1]=2 \delta_{K}^{+}[2]
$$

and

$$
\sigma_{K}=\delta_{K}^{-}[2]-\delta_{K}^{-}[1], \quad w_{K}=2 \delta_{K}^{-}[2]
$$




\section{Computing the Jones slopes and the Jones period of a knot}

4.1. Some lemmas on quasi-polynomials. To better present the experimental (and in some cases, proven) data presented in the next section, let us give some lemmas on quasi-polynomials. If $a(n)$ is a sequence of numbers, consider the generating series

$$
G_{a}(z)=\sum_{n=0}^{\infty} a(n) z^{n} .
$$

The next well-known lemma characterizes quasi-polynomials. It appears in [St], Prop. 4.4.1, and [BR], Lemma 3.24.

Lemma 4.1 ([St], Prop. 4.4.1, [BR], Lemma 3.24). The following are equivalent:

(a) a (n) is a quasi-polynomial of period $\pi$.

(b) The generating series

$$
G_{a}(z)=\frac{P(z)}{Q(z)}
$$

is a rational function where $P(z), Q(z) \in \mathbb{C}[z]$, every zero $\alpha$ of $Q(z)$ satisfies $\alpha^{\pi}=1$ (provided that $P(z) / Q(z)$ has been reduced to lowest term) and $\operatorname{deg} P<\operatorname{deg} Q$,

(c) For all $n \geq 0$,

$$
a(n)=\sum_{i=1}^{k} p_{i}(n) \gamma_{i}^{n}
$$

where each $p_{i}(n)$ is a polynomial function of $n$ and each $\gamma_{i}$ satisfies $\gamma_{i}^{\pi}=1$.

Moreover, the degree of $p_{i}(n)$ in (22) is one less than the multiplicity of the root $\gamma_{i}^{-1}$ in $Q(z)$, provided $P(z) / Q(z)$ has been reduced to lowest terms.

Definition 4.2. We say that a quadratic quasi-polynomial $a(n)$ is mono-sloped if it has only one slope $s$. In other words, we have

$$
a(n)=\frac{s}{2} n^{2}+b(n),
$$

where $b(n)$ is a linear quasi-polynomial.

To phrase our next corollary, let $\Phi_{n}(z)$ denote the $n$-th cyclotomic polynomial, and let $\phi(n)$ denote Euler's $\phi$-function.

Corollary 4.3. $a(n)$ is mono-sloped if and only if

$$
G_{a}(z)=\frac{a z^{2}+b z+c}{(1-z)^{3}}+\sum_{d>1} \frac{R_{d}(z)}{\Phi_{d}(z)^{c_{d}}},
$$


where the summation is over a finite set of natural numbers and $c_{p} \leq 2$ and $R_{d}(z) \in$ $\mathbb{C}[z]$ has degree less than $\phi(d) c_{d}$. Moreover,

$$
s=a+b+c .
$$

Proof. Observe that

$$
\sum_{n=0}^{\infty}\left(\alpha n^{2}+\beta n+\gamma\right) z^{n}=\frac{a z^{2}+b z+c}{(1-z)^{3}}
$$

if and only if

$$
\alpha=\frac{1}{2}(a+b+c), \quad \beta=\frac{1}{2}(-a+b+3 c), \quad \gamma=c .
$$

Proof of Lemma 1.10. Given $s$, there exists an arithmetic progression $\pi n+k$ such that for all natural numbers $n$ we have

$$
a(\pi n+k)=\frac{s}{2}(\pi n+k)^{2}+\beta(\pi n+k)+\gamma .
$$

Now let

$$
\begin{aligned}
b(n) & =\frac{s}{2}(\pi n+k)^{2}+\beta(\pi n+k)+\gamma \\
& =s \pi^{2}\left(\begin{array}{l}
n \\
2
\end{array}\right)+\left(\beta \pi+k \pi s+\frac{\pi^{2} s}{2}\right) n+\gamma+\beta k+\frac{k^{2} s}{2} .
\end{aligned}
$$

Now $b$ takes integer values at all integers. This implies that

$$
s \pi^{2} \in \mathbb{Z}, \quad \beta \pi+k \pi s+\frac{\pi^{2} s}{2} \in \mathbb{Z}, \quad \gamma+\beta k+\frac{k^{2} s}{2} \in \mathbb{Z}
$$

(see $[\mathrm{BR}])$. The result follows.

It is often easier to detect the periodicity properties of the difference

$$
(\Delta a)(n):=a(n+1)-a(n)
$$

of a sequence $a(n)$. It is easy to recover $G_{a}(z)$ from $G_{\Delta a}(z)$ and $G_{a}(0)$.

Lemma 4.4. With the above conventions, we have

$$
G_{\Delta a}(z)=\frac{G_{a}(z)(1-z)-G_{a}(0)}{z} .
$$

Proof. We have

$$
\begin{aligned}
G_{\Delta a}(z) & =\sum_{n=0}^{\infty}(a(n+1)-a(n)) z^{n} \\
& =\frac{1}{z} \sum_{n=0}^{\infty} a(n+1) z^{n+1}-\sum_{n=0}^{\infty} a(n) z^{n} \\
& =\frac{1}{z}\left(G_{a}(z)-G_{a}(0)\right)-G_{a}(z)
\end{aligned}
$$


We can iterate the above by considering the $k$-th difference defined by $\Delta^{0} a=a$ and $\Delta^{k} a=\Delta\left(\Delta^{k-1} a\right)$ for $k \geq 1$.

4.2. Computing the colored Jones function of a knot. There are several ways to compute the colored Jones function $J_{K, n}(q)$ of a knot $K$. For example, one may use a planar projection and $R$-matrices; see for example, [Tu2], [GL1] and also [B-N]). Alternatively, one may use planar projections and shadow formulas as discussed at length in [Co] and [GVa]. Or one may use fusion quantum spin networks and recoupling theory, discussed in [CFS], [KL], [Co], [GVa]. All these approaches gives various useful formulas for $J_{K, n}(q)$ presented as a finite sum of a proper $q$ hypergeometric summand [GL1]. A careful inspection of the summand allows in several cases to compute the degree of $J_{K, n}(q)$.

4.3. Guessing the colored Jones function of a knot. In this section we guess the sequence $\delta_{K}$ of knots with a small number of crossings, using the following strategy, inspired by conversations with D. Zagier. Using the Knot Atlas [B-N] we compute as many values $J_{K, n}(q)$ of the colored Jones function as we can, and record their degree. This gives us a table of values of the quadratic quasi-polynomials $\delta_{K}(n)$ and $\delta_{K}^{*}(n)$. Taking the third difference of this table results into a degree 0 quasi-polynomial, i.e., a periodic function. At this point, we make a guess for this periodic function, and the corresponding generating series. Then, we use Lemma 4.4 and our guess for the second different to obtain a formula for $G_{\delta_{K}}(z)$ and $G_{\delta_{K}^{*}}(z)$. The partial fraction decomposition then gives us a formula for $\delta_{K}(n)$ and $\delta_{K}^{*}(n)$. In some cases, using explicit finite multi-dimensional sum formulas for the colored Jones polynomial, one can prove that the guessed formula for $\delta_{K}(n)$ and $\delta_{K}^{*}(n)$ are indeed correct. In this section, we will not bother with proofs.

As an example of our method, we will guess a formula for $\delta(n)$ and $\delta^{*}(n)$ for the $(-2,3,7)$ pretzel knot. One can actually prove that our guess is correct, using the fusion formulas for the 3-pretzel knots, but we will not bother. The values of $\delta^{*}(n)$ starting with $n=0$ are given by:

$$
\delta^{*}: 0,5,10,15,20,25,30,35,40,45,50,55,60,65,70, \ldots
$$

Taking the first difference we get the following values of $\left(\Delta \delta^{*}\right)(n)$ starting with $n=0$ :

$$
\Delta \delta^{*}: 5,5,5,5,5,5,5,5,5,5,5,5,5,5, \ldots
$$

appears to be the constant sequence from which we guess that $\delta^{*}(n)=5 n$, and correspondingly the generating series is

$$
G_{\delta^{*}}(z)=\frac{5 z}{(1-z)^{2}} .
$$


More interesting is the sequence $\delta(n)$ starting with $n=0$ :

$\delta: 0,13,35,67,108,158,217,286,364,451,547,653,768,892$, $1025,1168,1320,1481,1651,1831, \ldots$

Taking the first, second and third difference we obtain

$\Delta \delta: 13,22,32,41,50,59,69,78,87,96,106,115,124,133,143$, $152,161,170,180, \ldots$,

$\Delta^{2} \delta: 9,10,9,9,9,10,9,9,9,10,9,9,9,10,9,9,9,10, \ldots$,

$\Delta^{3} \delta: 1,-1,0,0,1,-1,0,0,1,-1,0,0,1,-1,0,0,1, \ldots$

Thus, we guess that $\Delta^{3} \delta$ is a periodic sequence with period 4 and generating series

$$
G_{\Delta^{3} \delta}(z)=\sum_{n=0}^{\infty}\left(z^{4 n}-z^{4 n+1}\right)=\frac{1}{(1+z)\left(1+z^{2}\right)} .
$$

Using Lemma 4.4 three times, we compute

$$
\begin{aligned}
G_{\delta}(z) & =\frac{13 z+9 z^{2}+10 z^{3}+9 z^{4}-4 z^{5}}{(1-z)^{3}\left(1+z+z^{2}+z^{3}\right)} \\
& =\frac{-3+216 z-65 z^{2}}{16(1-z)^{3}}+\frac{3+4 z-z^{2}}{16\left(1+z+z^{2}+z^{3}\right)} .
\end{aligned}
$$

Taking the partial fraction decomposition, it follows that

$$
\delta(n)=\left[\frac{37}{8} n^{2}+\frac{17}{2} n\right]=\frac{37}{8} n^{2}+\frac{17}{2} n+\varepsilon(n),
$$

where $\varepsilon(n)$ is a periodic sequence of period 4 given by

$$
\varepsilon(n)= \begin{cases}0 & \text { if } n=0 \bmod 4 \\ \frac{1}{8} & \text { if } n=1 \bmod 4 \\ \frac{1}{2} & \text { if } n=2 \bmod 4 \\ \frac{1}{8} & \text { if } n=3 \bmod 4\end{cases}
$$

4.4. A summary of non-alternating knots. In this section we list the quasi-polynomials $\delta_{K}$ and $\delta_{K}^{*}$ of non-alternating knots $K$ with 8 and 9 crossings. In the Rolfsen table of knots, the non-alternating knots with 8 crossings are $8_{k}$, where $k=19, \ldots, 21$, and the non-alternating knots with 9 crossings are $9_{k}$, where $k=42, \ldots, 49$. Let us give a combined table of the non-alternating knots $K$ with 8 and 9 crossings, their periods $\pi_{K}$, their Jones slopes $\mathrm{js}_{K}$ and $\mathrm{js}_{K}^{*}$, and their distinct boundary slopes. The boundary slopes $\mathrm{bs}_{K}$ are computed using the program of [HO], corrected in [Du], 


\begin{tabular}{|c|c|c|c|c|}
\hline$K$ & $\pi_{K}$ & $\mathrm{js}_{K}$ & $\mathrm{js}_{K}^{*}$ & $\mathrm{bs}_{K}$ \\
\hline $8_{19}$ & 2 & 6 & 0 & $\{0,12\}$ \\
$8_{20}$ & 3 & $4 / 3$ & -5 & $\{-10,0,8 / 3\}$ \\
$8_{21}$ & 2 & $1 / 2$ & -6 & $\{-12,-6,-2,0,1\}$ \\
\hline $9_{42}$ & 2 & 3 & -4 & $\{-8,0,8 / 3,6\}$ \\
$9_{43}$ & 3 & $16 / 3$ & -2 & $\{-4,0,6,8,32 / 3\}$ \\
$9_{44}$ & 3 & $7 / 3$ & -5 & $\{-10,-2,0,1,2,14 / 3\}$ \\
$9_{45}$ & 2 & $1 / 2$ & -7 & $\{-14,-10,-8,-4,-2,0,1\}$ \\
$9_{46}$ & 2 & 1 & -6 & $\{-12,0,2\}$ \\
$9_{47}$ & 2 & $9 / 2$ & -3 & $\{-6,0,4,8,9,16\}$ \\
$9_{48}$ & 2 & $11 / 2$ & -2 & $\{-4,0,4,8,11\}$ \\
$9_{49}$ & 2 & $15 / 2$ & 0 & $\{0,4,6,12,15\}$ \\
\hline
\end{tabular}

which computes the boundary slopes of all Montesinos knots except 949 which is not a Montesinos knots. In all those cases, the set of boundary slopes agrees with the slopes of the $A$-polynomial of [Cu], [CCGLS], once 0 is included.

The above data are in agreement with Conjecture 1. Let us make a phenomenological remark regarding all examples of non-alternating knots with 8 or 9 crossings.

(a) $\delta^{*}(n)$ and $\delta(n)$ are mono-sloped, i.e., they are of the form $s n^{2} / 2+\varepsilon(n)$ where $\varepsilon(n)$ is a linear quasi-polynomial.

(b) For all knots, $2 \mathrm{js}$ is a boundary slope, though not necessarily the largest one.

(c) In the case of the $8_{20}, 9_{43}$ and $9_{44}$ knots the degree of $\varepsilon(n)$ is 1 , and in all other cases it is zero.

(d) The period of all non-alternating knots is greater than 1 . The $8_{20}, 9_{43}, 9_{44}$ knots have period 3 , and $(-2,3,7)$ has period 4 . The period of $(-2,3, p)$ for odd $p \geq 5$ appears to be $p-3$, and the number of crossings is $p+5$. Thus the period can be asymptotically as large as the number of crossings.

(e) For the cases of $8_{21}, 9_{45}, 9_{46}, 9_{47}$ the leading coefficient is 2 , and for $9_{48}, 9_{49}$ it is -2 .

\subsection{The 8-crossing non-alternating knots}

4.5.1. The $8_{19}$ knot. In the data below, we will give the first few values of $\delta^{*}(n)$ and $\delta(n)$, the guessed decomposition of the generating series $G_{\delta^{*}}(z)$ and $G_{\delta}(z)$ of the quasi-polynomials $\delta^{*}$ and $\delta$. 
Some values of $\delta^{*}(n)$ and $\delta(n)$ starting with $n=0$ :

$$
\begin{aligned}
\delta^{*}: 0,3,6,9,12,15,18,21, \ldots, & \\
& \delta: 0,8,23,43,70,102,141,185, \ldots, \\
G_{\delta^{*}}(z)= & \frac{3 z}{(1-z)^{2}}, \\
G_{\delta}(z)= & \frac{8 z+7 z^{2}-3 z^{3}}{(1-z)^{3}(1+z)}=\frac{-1+36 z-11 z^{2}}{4(1-z)^{3}}+\frac{1}{4(1+z)}, \\
\delta^{*}(n)= & 3 n, \\
\delta(n)= & 3 n^{2}+\frac{11}{2} n-\frac{1}{4}+\varepsilon(n),
\end{aligned}
$$

where $\varepsilon(n)=(-1)^{n} / 4$ is a 2-periodic sequence. Note that in this example the values of $\delta_{K}^{*}(n)$ for $n=0,1,2,3$ suffice to prove that $\delta_{K}(n)$ is not a polynomial of $n$.

\subsubsection{The $8_{20}$ knot}

$$
\begin{aligned}
\delta^{*}: & 0,-5,-15,-30,-50,-75,-105,-140,-180,-225,-275,-330, \\
& -390,-455,-525,-600,-680,-765,-855,-950,-1050, \ldots, \\
\delta: & 0,1,2,7,12,16,26,35,42,57,70,80,100,117,130,155,176, \\
& 192,222,247,266, \ldots,
\end{aligned}
$$

$$
\begin{aligned}
G_{\delta^{*}}(z) & =-\frac{5 z}{(1-z)^{3}}, \\
G_{\delta}(z) & =\frac{z+z^{2}+5 z^{3}+3 z^{4}+2 z^{5}}{(1-z)^{3}\left(1+z+z^{2}\right)^{2}} \\
& =\frac{-2+12 z+2 z^{2}}{9(1-z)^{3}}+\frac{2+7 z+4 z^{2}+2 z^{3}}{9\left(1+z+z^{2}\right)^{2}}, \\
\delta^{*}(n) & =-\frac{5 n(n+1)}{2}, \\
\delta(n) & =\frac{2}{3} n^{2}+\frac{2}{9} n-\frac{2}{9}+\varepsilon(n),
\end{aligned}
$$

where $\varepsilon(n)$ is a linear quasi-polynomial with period 3 .

\subsubsection{The $8_{21}$ knot}

$$
\begin{aligned}
& \delta^{*}: 0,-7,-20,-39,-64,-95,-132,-175, \ldots, \\
& \delta: 0,-1,-1,-1,0,1,3,5, \ldots,
\end{aligned}
$$




$$
\begin{aligned}
G_{\delta^{*}}(z) & =\frac{-7 z+z^{2}}{(1-z)^{3}}, \\
G_{\delta}(z) & =\frac{-z+z^{2}+z^{3}}{(1-z)^{3}(1+z)}=\frac{-1-4 z+9 z^{2}}{8(1-z)^{3}}+\frac{1}{8(1+z)}, \\
\delta^{*}(n) & =-n(3 n+4), \\
\delta(n) & =\frac{1}{4} n^{2}-n-\frac{1}{8}-\varepsilon(n),
\end{aligned}
$$

where $\varepsilon(n)=(-1)^{n} / 8$ is a 2-periodic sequence.

\subsection{The 9-crossing non-alternating knots}

\subsubsection{The $9_{42}$ knot}

$$
\begin{aligned}
\delta^{*}: & 0,-3,-10,-21,-36,-55,-78,-105, \ldots, \\
\delta: & 0,3,10,19,32,47,66,87, \ldots, \\
G_{\delta^{*}}(z) & =\frac{-3 z-z^{2}}{(1-z)^{3}}, \\
G_{\delta}(z) & =\frac{z\left(3+4 z-z^{2}\right)}{(1-z)^{3}(1+z)}=\frac{1-16 z+3 z^{2}}{4(-1+z)^{3}}+\frac{1}{4(1+z)}, \\
\delta^{*}(n) & =-n(2 n+1), \\
\delta(n) & =\frac{3}{2} n^{2}+2 n-\frac{1}{4}+\varepsilon(n),
\end{aligned}
$$

where $\varepsilon(n)=(-1)^{n} / 4$ is a 2-periodic sequence.

\subsubsection{The $9_{43}$ knot}

$$
\begin{aligned}
& \delta^{*}: 0,0,-2,-6,-12,-20,-30,-42, \ldots, \\
& \quad \delta: 0,7,17,37,60,85,122,161, \ldots,
\end{aligned}
$$

$$
\begin{aligned}
G_{\delta^{*}}(z)= & (2 z) /(-1+z)^{3}, \\
G_{\delta}(z)= & \left(z\left(-7-10 z-20 z^{2}-9 z^{3}-5 z^{4}+3 z^{5}\right)\right) /\left((-1+z)^{3}\left(1+z+z^{2}\right)^{2}\right) \\
= & \left(5-72 z+19 z^{2}\right) /\left(9(-1+z)^{3}\right) \\
& +\left(5+16 z+13 z^{2}+8 z^{3}\right) /\left(9\left(1+z+z^{2}\right)^{2}\right), \\
\delta^{*}(n)= & -n(n-1), \\
\delta(n)= & -(5 / 9)+(38 n) / 9+\left(8 n^{2}\right) / 3+\varepsilon(n),
\end{aligned}
$$

where $\varepsilon(n)$ is a linear quasi-polynomial with period 3 . 


\subsubsection{The $9_{44}$ knot}

$$
\begin{aligned}
& \delta^{*}: 0,-5,-15,-30,-50,-75,-105,-140, \ldots, \\
& \delta: 0,2,5,13,22,31,47,63, \ldots, \\
G_{\delta^{*}}(z)= & (5 z) /(-1+z)^{3}, \\
G_{\delta}(z)= & -\left(\left(z\left(2+3 z+8 z^{2}+5 z^{3}+3 z^{4}\right)\right) /\left((-1+z)^{3}\left(1+z+z^{2}\right)^{2}\right)\right) \\
= & \left(2-21 z-2 z^{2}\right) /\left(9(-1+z)^{3}\right) \\
& +\left(2+7 z+4 z^{2}+2 z^{3}\right) /\left(9\left(1+z+z^{2}\right)^{2}\right), \\
\delta^{*}(n)= & -5 n(n+1) / 2, \\
\delta(n)=- & (2 / 9)+(13 n) / 18+\left(7 n^{2}\right) / 6+\varepsilon(n),
\end{aligned}
$$

where $\varepsilon(n)$ is a linear quasi-polynomial with period 3 .

\subsubsection{The $9_{45}$ knot}

$$
\begin{aligned}
\delta^{*}: 0 & ,-8,-23,-45,-74,-110,-153,-203, \ldots, \\
\delta: 0 & -1,-1,-1,0,1,3,5, \ldots, \\
G_{\delta^{*}}(z) & =-\left(\left(-8 z+z^{2}\right) /(-1+z)^{3}\right), \\
G_{\delta}(z) & =-\left(\left(z\left(-1+z+z^{2}\right)\right) /\left((-1+z)^{3}(1+z)\right)\right) \\
& =\left(1+4 z-9 z^{2}\right) /\left(8(-1+z)^{3}\right)+1 /(8(1+z)), \\
\delta^{*}(n) & =-n(7 n+9) / 2, \\
\delta(n) & =-(1 / 8)-n+n^{2} / 4+\varepsilon(n),
\end{aligned}
$$

where $\varepsilon(n)=(-1)^{n} / 8$ is a periodic sequence with period 2 .

\subsubsection{The $9_{46}$ knot}

$$
\begin{aligned}
\delta^{*}: 0,-6,-18, & -36,-60,-90,-126,-168, \ldots, \\
\delta: 0,0,2,4, & , 12,18,24, \ldots, \\
G_{\delta^{*}}(z) & =-\frac{6 z}{(1-z)^{3}}, \\
G_{\delta}(z) & =\frac{2 z^{2}}{(1-z)^{3}(1+z)} \\
& =\frac{-1+4 z+z^{2}}{4(1-z)^{3}}+\frac{1}{4(1+z)}, \\
\delta^{*}(n) & =-3 n(n+1), \\
\delta(n) & =-(1 / 4)+n^{2} / 2+\varepsilon(n),
\end{aligned}
$$


where $\varepsilon(n)=(-1)^{n} / 4$ is a periodic sequence with period 2 .

\subsubsection{The $9_{47}$ knot}

$$
\begin{aligned}
\delta^{*} & : 0,-2,-7,-15,-26,-40,-57,-77, \ldots, \\
\delta & : 0,5,15,29,48,71,99,131, \ldots, \\
G_{\delta^{*}}(z) & =\left(2 z+z^{2}\right) /(-1+z)^{3}, \\
G_{\delta}(z) & =\left(z\left(-5-5 z+z^{2}\right)\right) /\left((-1+z)^{3}(1+z)\right) \\
& =\left(1-44 z+7 z^{2}\right) /\left(8(-1+z)^{3}\right)+1 /(8(1+z)), \\
\delta^{*}(n) & =-n(3 n+1) / 2, \\
\delta(n) & =-(1 / 8)+3 n+\left(9 n^{2}\right) / 4+\varepsilon(n),
\end{aligned}
$$

where $\varepsilon(n)=(-1)^{n} / 8$ is a periodic sequence with period 2 .

\subsubsection{The $9_{48}$ knot}

$$
\begin{aligned}
& \delta^{*}: 0,-1,-4,-9,-16,-25, \ldots, \\
& \delta: 0,6,18,35,58,86, \ldots, \\
& G_{\delta^{*}}(z)=-\left(\left(-z-z^{2}\right) /(-1+z)^{3}\right), \\
& G_{\delta}(z)=\left(z\left(-6-6 z+z^{2}\right)\right) /\left((-1+z)^{3}(1+z)\right) \\
&=\left(1-52 z+7 z^{2}\right) /\left(8(-1+z)^{3}\right)+1 /(8(1+z)), \\
& \delta^{*}(n)=- n^{2}, \\
& \delta(n)=-(1 / 8)+(7 n) / 2+\left(11 n^{2}\right) / 4+\varepsilon(n),
\end{aligned}
$$

where $\varepsilon(n)=(-1)^{n} / 8$ is a periodic sequence with period 2 .

\subsubsection{The $9_{49}$ knot}

$$
\begin{gathered}
\delta^{*}: 0,2,4,6,8,10, \ldots, \\
\delta: 0,9,26,50,82,121, \ldots, \\
G_{\delta^{*}}(z)=(2 z) /(-1+z)^{2}, \\
G_{\delta}(z)=\left(z\left(-9-8 z+2 z^{2}\right)\right) /\left((-1+z)^{3}(1+z)\right) \\
=\left(1-76 z+15 z^{2}\right) /\left(8(-1+z)^{3}\right)+1 /(8(1+z)), \\
\delta^{*}(n)=2 n, \\
\delta(n)=-(1 / 8)+(11 n) / 2+\left(15 n^{2}\right) / 4+\varepsilon(n),
\end{gathered}
$$

where $\varepsilon(n)=(-1)^{n} / 8$ is a periodic sequence with period 2 . 
4.7. The case of the $(-2,3, p)$ pretzel knots. A triple sum formula for the colored Jones polynomial of pretzel knots with 3 pretzels is available, and using it we can compute $\delta_{K}(n)$ and $\delta_{K}^{*}(n)$ for all pretzel knots of the form $(-2,3, p)$ for odd $p$; see [GVa]. We will state the result of the computation here. Recall the $k$-th difference $\Delta^{k} f$ of a sequence $f$ from Section 4.1. When $p>0$ is odd, we have

$$
G_{\delta^{*}}(z)=\frac{(p+3) z}{2(1-z)^{2}}, \quad G_{\Delta^{3} \delta}(z)= \begin{cases}\frac{z^{p-7}(1-z)}{1-z^{p-3}}, & p \geq 7 \\ -\frac{3}{1+z}, & p=5 \\ -\frac{2}{1+z}, & p=3 \\ 0 & p=1\end{cases}
$$

It follows that

$$
\delta^{*}(n)=\frac{(p+3) n}{2}, \quad \delta(n)= \begin{cases}\frac{5}{2} n^{2}+\varepsilon_{p}(n), & p=1, \\ 3 n^{2}+\varepsilon_{p}(n), & p=3, \\ \frac{\left(p^{2}-p-5\right) n^{2}}{2(p-3)}+\varepsilon_{p}(n), & p \geq 5,\end{cases}
$$

where $\varepsilon_{p}(n)$ are linear quasi-polynomials. When $p<0$ is odd we have

$$
\begin{aligned}
\Delta^{3} G_{\delta^{*}}(z) & = \begin{cases}0, & p=-1, \\
-\frac{4+4 z+3 z^{2}+z^{3}}{\left(1+z+z^{2}\right)^{2}}, & p=-3, \\
\frac{z^{|p|-4}-2 z^{|p|-3}-\sum_{k=|p|-2}^{2|p|-4} z^{k}}{\left(\sum_{k=0}^{|p|-1} z^{k}\right)^{2}}, & p \leq-5,\end{cases} \\
G_{\delta}(z) & =\frac{z(p+13-(p+3) z)}{2(1-z)^{3}} .
\end{aligned}
$$

It follows that

$$
\delta^{*}(n)=\left\{\begin{array}{ll}
\frac{5}{2} n^{2}+\varepsilon_{p}(n), & p=-1, \\
\frac{(p+1)^{2} n^{2}}{2 p}+\varepsilon_{p}(n), & p \leq-3,
\end{array} \quad \delta(n)=\frac{n(5 n+(p+8))}{2},\right.
$$

where $\varepsilon_{p}(n)$ are linear quasi-polynomials. Notice that the above formulas single out exceptional behavior at $p=-3,-1,1,3,5$. The Jones period and the Jones slopes are given by

$$
\pi=\left\{\begin{array}{ll}
p-3, & p \geq 5, \\
2, & p=3, \\
|p|, & p \leq 1,
\end{array} \quad \mathrm{js}=\left\{\begin{array}{ll}
\frac{p^{2}-p-5}{p-3}, & p \geq 5, \\
6, & p=3, \\
5, & p \leq-1,
\end{array} \quad \mathrm{js} *= \begin{cases}0, & p \geq 5 \\
0, & p=3 \\
\frac{(p+1)^{2}}{p}, & p \leq 1\end{cases}\right.\right.
$$

On the other hand, Hatcher-Oertel and Dunfield (see [HO] and [Du]) compute the slopes of those Montesinos knots:

$$
\mathrm{bs}_{p}= \begin{cases}\left\{0,16, \frac{2\left(p^{2}-p-5\right)}{p-3}, 2(3+p)\right\}, & p \geq 7 \\ \left\{0,10,2 \frac{(p+1)^{2}}{p}, 2(p+3)\right\}, & p \leq-1\end{cases}
$$


Compare also with Mattman [Ma], p. 32, who computes which of those slopes are visible from the geometric component of the $A$-polynomial. Equations (24) and (25) together with the fact that 0 is a boundary slope confirm Conjecture 1 for all $(-2,3, p)$ pretzel knots.

4.8. The case of torus knots. In this section we will use Morton's formula for the colored Jones function of a torus knot to compute the degree of the colored Jones function and verify Conjecture 1 .

Let $T(a, b)$ denote the $(a, b)$ torus knot for a pair of coprime integers $a, b$. Since the mirror image of $T(a, b)$ is $T(a,-b)$, we will focus on the case of $a, b>0$. With our conventions, Morton's formula [Mo] for the colored Jones function is the following:

$$
J_{T(a, b), n}(q)=\frac{q^{\frac{1}{4} a b n(n+2)}}{q^{\frac{n+1}{2}}-q^{-\frac{n+1}{2}}} \sum_{k=-\frac{n}{2}}^{\frac{n}{2}}\left(q^{-a b k^{2}+(a-b) k+\frac{1}{2}}-q^{-a b k^{2}+(a+b) k-\frac{1}{2}}\right) .
$$

For example,

$$
\begin{aligned}
& J_{T(2,3), 1}(q)=q+q^{3}-q^{4}, \\
& J_{T(3,4), 2}(q)=q^{6}+q^{9}+q^{12}-q^{13}-q^{16}-q^{19}+q^{20}-q^{22}+q^{23} .
\end{aligned}
$$

The summand of equation (26) consists of two monomials with exponents quadratic functions of $k$. A little calculation reveals that the maximum and minimum degree of the colored Jones function is given by

$$
\begin{aligned}
& \delta_{T(a, b)}(n)=\frac{a b}{4} n^{2}+\frac{a b-1}{2} n-\left(1-(-1)^{n}\right) \frac{(a-2)(b-2)}{8}, \\
& \delta_{T(a, b)}^{*}(n)=\frac{(a-1)(b-1)}{2} n .
\end{aligned}
$$

Thus the period $\pi_{T(a, b)}$ is 2 when $a, b \neq 2$ and 1 when $a=2$ or $b=2$. The boundary slopes of $T(a, b)$ are $\{0, a b\}$ (see for example [HO]). This confirms Conjecture 1 for torus knots.

Acknowledgment. The author wishes to thank K. Ichihara, Y. Kabaya, T. Mattman, P. Ozsváth, A. Shumakovitch, R. van der Veen, D. Zagier, D. Zeilberger and especially M. Culler and N. Dunfield for numerous enlightening conversations. The idea of the slope conjecture was conceived during the New York Conference on Interactions between Hyperbolic Geometry, Quantum Topology and Number Theory in New York in the summer of 2009. The author wishes to thank the organizers, A. Champanerkar, O. Dasbach, E. Kalfagianni, I. Kofman, W. D. Neumann and N. Stoltzfus for their hospitality. 


\section{References}

[B-N] Bar-Natan, The knot atlas. http://katlas.math.toronto.edu/wiki/Main_Page

[B-NG] D. Bar-Natan and S. Garoufalidis, On the Melvin-Morton-Rozansky conjecture. Invent. Math. 125 (1996), 103-133. Zbl 0855.57004 MR 1389962

[B-NL] D. Bar-Natan and R. Lawrence, A rational surgery formula for the LMO invariant. Israel J. Math. 140 (2004), 29-60. Zbl 1062.57015 MR 2054838

[BP] A. Barvinok and J. E. Pommersheim, An algorithmic theory of lattice points in polyhedra. In New perspectives in algebraic combinatorics (Berkeley, CA, 199697), Math. Sci. Res. Inst. Publ. 38, Cambridge University Press, Cambridge 1999, 91-147. Zbl 0940.05004 MR 1731815

[BR] M. Beck and S. Robins, Computing the continuous discretely. Undergrad. Texts Math., Springer-Verlag, New York 2007. Zbl 1114.52013 MR 2271992

[BV] M. Brion and M. Vergne, Lattice points in simple polytopes. J. Amer. Math. Soc. 10 (1997), 371-392. Zbl 0871.52009 MR 1415319

$[\mathrm{Bu}] \quad$ B. A. Burton, Introducing Regina, the 3-manifold topology software. Experiment. Math. 13 (2004), 267-272. Zbl 1090.57003 MR 2103324

[CFS] S. Carter, D. E. Flath, and M. Saito, The classical and quantum $6 j$-symbols. Math. Notes 43, Princeton University Press, Princeton, NJ, 1995. Zbl 0851.17001 MR 1366832

[CCGLS] D. Cooper, M. Culler, H. Gillet, D. D. Long, and P. B. Shalen, Plane curves associated to character varieties of 3-manifolds. Invent. Math. 118 (1994), 47-84. Zbl 0842.57013 MR 1288467

[Co] F. Costantino, Integrality of Kauffman brackets of trivalent graphs. Preprint 2009. arXiv:0908.0542 [math.QA]

[Cu] M. Culler, Tables of $A$-polynomials. Available at http://www.math.uic.edu/ culler

[CGLS] M. Culler, C. M. Gordon, J. Luecke, and P. B. Shalen, Dehn surgery on knots. Ann. of Math. (2) 125 (1987), 237-300. Zbl 0633.57006 MR 881270

[CS] M. Culler and P. B. Shalen, Bounded, separating, incompressible surfaces in knot manifolds. Invent. Math. 75 (1984), 537-545. Zbl 0542.57011 MR 735339

[CT] C. L. Curtis and S. Taylor, The Jones polynomial and boundary slopes of alternating knots. Preprint 2009, arXiv:0910.4912 [math.GT]

[Du] N. M. Dunfield, A table of boundary slopes of Montesinos knots. Topology 40 (2001), 309-315. Zbl 0967.57014 MR 1808223

[DG] N. M. Dunfield and S. Garoufalidis, Boundary slopes of 1-cusped manifolds. Preprint 2010.

[Eh] E. Ehrhart, Sur les polyèdres homothétiques bordés à $n$ dimensions. C. R. Acad. Sci. Paris 254 (1962), 988-990. Zbl 0100.27602 MR 0131403

[FKP] D. Futer, E. Kalfagianni, and J. S. Purcell, Dehn filling, volume, and the Jones polynomial. J. Differential Geom. 78 (2008), 429-464. Zbl 1144.57014 MR 2396249

[Ga1] S. Garoufalidis, On the characteristic and deformation varieties of a knot. In Proceedings of the Casson Fest, Geom. Topol. Monogr. 7, Geom. Topol. Publ., Coventry 2004, 291-309. Zbl 1080.57014 MR 2172488 
[Ga2] S. Garoufalidis, The degree of a $q$-holonomic sequence is a quadratic quasipolynomial. Preprint 2010. arXiv:1005.4580 [math.CO]

[Ga3] S. Garoufalidis, Tropicalization and the slope conjecture for 2-fusion knots. Preprint 2010 .

[Ga4] S. Garoufalidis, Knots and tropical curves. Preprint 2010. arXiv:1003.4436 [math.GT]

[GL] S. Garoufalidis and Y. Lan, Experimental evidence for the volume conjecture for the simplest hyperbolic non-2-bridge knot. Algebr. Geom. Topol. 5 (2005), 379-403. Zbl 1092.57005 MR 2153123

[GL1] S. Garoufalidis and T. T. Q. Lê, The colored Jones function is $q$-holonomic. Geom. Topol. 9 (2005), 1253-1293. Zbl 1078.57012 MR 2174266

[GL2] S. Garoufalidis and T. T. Lê, Is the Jones polynomial of a knot really a polynomial? J. Knot Theory Ramifications 15 (2006), 983-1000. Zbl 1126.57007 MR 2275093

[GVa] S. Garoufalidis and R. van der Veen, Asymptotics of quantum spin networks at a fixed root of unity. Preprint 2010. arXiv:1003.4954 [math.GT]

[GVu] S. Garoufalidis and T. Vuong, The slope conjecture for simple Lie algebras. Preprint 2010.

[G-MM] J. González-Meneses and P. M. G. Manchón, A geometric characterization of the upper bound for the span of the Jones polynomial. Preprint 2009. arXiv:0907.5374 [math.GT]

[Ha] A. E. Hatcher, On the boundary curves of incompressible surfaces. Pacific J. Math. 99 (1982), 373-377. Zbl 0502.57005 MR 658066

[HaO] A. Hatcher and U. Oertel, Boundary slopes for Montesinos knots. Topology 28 (1989), 453-480. Zbl 0686.57006 MR 1030987

[HO] M. Hedden and P. Ording, The Ozsváth-Szabó and Rasmussen concordance invariants are not equal. Amer. J. Math. 130 (2008), 441-453. Zbl 1139.57012 MR 2405163

[IM1] K. Ichihara and S. Mizushima, Crossing number and diameter of boundary slope set of Montesinos knot. Comm. Anal. Geom. 16 (2008), 565-589. Zbl 1156.57004 MR 2429969

[IM2] K. Ichihara and S. Mizushima, Boundary slopes and the numbers of positive/negative crossings for Montesinos knots. Preprint 2008. arXiv:0809.4435 [math.GT]

[Ja] J. C. Jantzen, Lectures on quantum groups. Grad. Stud. Math. 6, Amer. Math. Soc., Providence, RI, 1996. Zbl 0842.17012 MR 1359532

[Jo] V. F. R. Jones, Hecke algebra representations of braid groups and link polynomials. Ann. of Math. (2) 126 (1987), 335-388. Zbl 0631.57005 MR 908150

[Ka] Y. Kabaya, Private communication.

[KR] E. Kang and J. H. Rubinstein, Ideal triangulations of 3-manifolds I: spun normal surface theory. In Proceedings of the Casson Fest, Geom. Topol. Monogr. 7, Geom. Topol. Publ., Coventry 2004, 235-265. Zbl 1085.57016 MR 2172486 
[Ks] R. M. Kashaev, The hyperbolic volume of knots from the quantum dilogarithm. Lett. Math. Phys. 39 (1997), 269-275. Zbl 0876.57007 MR 1434238

[Kf] L. H. Kauffman, State models and the Jones polynomial. Topology 26 (1987), 395407. Zbl 0622.57004 MR 899057

[KL] L. H. Kauffman and S. L. Lins, Temperley-Lieb recoupling theory and invariants of 3-manifolds. Ann. of Math. Stud. 134, Princeton University Press, Princeton, NJ, 1994. Zbl 0821.57003 MR 1280463

[Le] T. T. Q. Lê, The colored Jones polynomial and the $A$-polynomial of knots. $A d v$. Math. 207 (2006), 782-804. Zbl 1114.57014 MR 2271986

[Li] W. B. R. Lickorish, An introduction to knot theory. Grad. Texts in Math. 175, Springer-Verlag, New York 1997. Zbl 0886.57001 MR 1472978

[LT] W. B. R. Lickorish and M. B. Thistlethwaite, Some links with non-trivial polynomials and their crossing-numbers. Comment. Math. Helv. 63 (1988), 527-539. Zbl 0686.57002 MR 966948

[LTi] F. Luo and S. Tillmann, Angle structures and normal surfaces. Trans. Amer. Math. Soc. 360 (2008), 2849-2866. Zbl 1152.57004 MR 2379778

[Ma] T. W. Mattman, The Culler-Shalen seminorms of the $(-2,3, n)$ pretzel knot. J. Knot Theory Ramifications 11 (2002), 1251-1289. Zbl 1030.57011 MR 1949779

[MMR] T. W. Mattman, G. Maybrun, and K. Robinson, 2-bridge knot boundary slopes: diameter and genus. Osaka J. Math. 45 (2008), 471-489. Zbl 1147.57009 MR 2441951

[Mv] S. Matveev, Algorithmic topology and classification of 3-manifolds. 2nd ed., Algorithms Comput. Math. 9, Springer-Verlag, Berlin 2007. Zbl 1128.57001 MR 2341532

[Me] N. D. Mermin, Is the moon there when nobody looks? Reality and the quantum theory. Physics Today 38 (1985), no. 4, 38-47.

[Mo] H. R. Morton, The coloured Jones function and Alexander polynomial for torus knots. Math. Proc. Cambridge Philos. Soc. 117 (1995), 129-135. Zbl 0852.57007 MR 1297899

[MO] C. Manolescu and P. Ozsváth, On the Khovanov and knot Floer homologies of quasi-alternating links. In Proceedings of Gökova Geometry-Topology Conference 2007, Gökova Geometry/Topology Conference (GGT), Gökova 2008, 60-81. Zbl 1195.57032 MR 2509750

[Mu K. Murasugi, Jones polynomials and classical conjectures in knot theory. Topology 26 (1987), 187-194. Zbl 0628.57004 MR 895570

[OS] P. Ozsváth and Z. Szabó, On the Floer homology of plumbed three-manifolds. Geom. Topol. 7 (2003), 185-224. Zbl 1130.57302 MR 1988284

[St] R. P. Stanley, Enumerative combinatorics. Vol. 1, Cambridge Stud. Adv. Math. 49, Cambridge University Press, Cambridge 1997. Zbl 0889.05001 MR 1442260

[Th] M. B. Thistlethwaite, A spanning tree expansion of the Jones polynomial. Topology 26 (1987), 297-309. Zbl 0622.57003 MR 899051

[Tu1] V. G. Turaev, A simple proof of the Murasugi and Kauffman theorems on alternating links. Enseign. Math. (2) 33 (1987), 203-225. Zbl 0668.57009 MR 925987 
[Tu2] V. G. Turaev, The Yang-Baxter equation and invariants of links. Invent. Math. 92 (1988), 527-553. Zbl 0648.57003 MR 939474

[WZ] H. S. Wilf and D. Zeilberger, An algorithmic proof theory for hypergeometric (ordinary and " $q$ ") multisum/integral identities. Invent. Math. 108 (1992), 575-633. Zbl 0739.05007 MR 1163239

[Z] D. Zeilberger, A holonomic systems approach to special functions identities. $J$. Comput. Appl. Math. 32 (1990), 321-368. Zbl 0738.33001 MR 1090884

[Zh] J. Zhu, On Kauffman brackets. J. Knot Theory Ramifications 6 (1997), 125-148. Zbl 0878.57014 MR 1442185

Received February 4, 2010

S. Garoufalidis, School of Mathematics, Georgia Institute of Technology, Atlanta, GA 30332-0160, U.S.A.

E-mail: stavros@math.gatech.edu 